\title{
Review on the therapeutic activities of the genus Trichilia
}

\author{
Revisão sobre as atividades terapêuticas do gênero Trichilia \\ Revisión de las actividades terapéuticas del género Trichilia
}

Received: 04/10/2021 | Reviewed: 04/18/2021 | Accept: 04/22/2021 | Published: 04/27/2021

Lusinalva Leonardo da Silva

ORCID: https://orcid.org/0000-0002-5638-0204

Fluminense Federal University, Brazil

E-mail: lusinalvaleonardo@id.uff.br

Renata de Almeida

ORCID: https://orcid.org/0000-0002-4841-8954

Fluminense Federal University, Brazil

E-mail: realmeida@id.uff.br

Fabiana Texeira e Silva

ORCID: https://orcid.org/0000-0001-9217-7740

Fluminense Federal University, Brazil

E-mail: fateixeira@id.uff.br

Maurício Afonso Verícimo

ORCID: https://orcid.org/0000-0002-9240-2925

Fluminense Federal University, Brazil

E-mail: mavericimo@id.uff.br

\begin{abstract}
The genus Trichilia (Meliaceae family) has been attracting interest among Brazilian Flora, due to the biological activities of its secondary metabolites. This genus has species distributed throughout tropical America, recognized for its significant economic importance and high commercial value. Its composition consists mainly of terpenoids (triterpenes, sesquiterpenes, limonoids and steroids) and polyphenols (flavonoids and tannins). In folk medicine this genus is used in preparations such as antimicrobial, antimalarial, antiviral and antioxidant. Studies carried out with several species of the genus Trichilia show that these plants have antioxidant, anticholinesterase, antimicrobial activities against pathogens of great clinical importance, neuroprotective, anti-anaphylactic and anti-inflammatory. In addition, analgesic, antineoplasic, hepatoprotective and immunomodulatory activities have also been investigated and proven in the literature. Based on the great biotechnological potential of the genus Trichilia, from the few studies on its applications in human health and in view of the importance of searching for natural substances that have effective therapeutic activities, this review highlights the main therapeutic applications of species of this genus found in the literature, and stimulate further studies on the use of these plants in the treatment of various diseases. For this, a survey on the topic was carried out in the main sources of scientific research in the period from 2005 to 2020, leading us to conclude that this genus can be a potent ally in the search for new phytotherapic drugs.
\end{abstract}

Keywords: Genus Trichilia; Meliaceae family; Treatment; Diseases; Medicinal plants.

\section{Resumo}

O gênero Trichilia (família Meliaceae) vem despertando interesse na Flora Brasileira, devido às atividades biológicas de seus metabólitos secundários. Este gênero possui espécies distribuídas por toda a América tropical, reconhecidas por sua significativa importância econômica e alto valor comercial. Sua composição consiste principalmente em terpenóides (triterpenos, sesquiterpenos, limonóides e esteróides) e polifenóis (flavonóides e taninos). Na medicina popular, este gênero é usado em preparações como antimicrobianos, antimaláricos, antivirais e antioxidantes. Estudos realizados com várias espécies do gênero Trichilia mostram que essas plantas possuem atividades antioxidantes, anticolinesterásicas, antimicrobianas contra patógenos de grande importância clínica, neuroprotetoras, antianafiláticas e antiinflamatórias. Além disso, as atividades analgésicas, antineoplásicas, hepatoprotetoras e imunomoduladoras também foram investigadas e comprovadas na literatura. Com base no grande potencial biotecnológico do gênero Trichilia, diante dos poucos estudos sobre suas aplicações na saúde humana e tendo em vista a importância da busca por substâncias naturais que tenham atividades terapêuticas eficazes, esta revisão destaca as principais aplicações terapêuticas das espécies deste gênero encontradas na literatura, e estimular novos estudos sobre a utilização dessas plantas no tratamento de diversas doenças. Para isso, foi realizado um levantamento sobre o tema nas principais fontes de pesquisas científicas no período de 2005 a 2020, levando-nos a concluir que esse gênero pode ser um potente aliado na busca por novos fitoterápicos.

Palavras-chave: Gênero Trichilia; Família Meliaceae; Tratamento; Doenças; Plantas medicinais. 


\begin{abstract}
Resumen
El género Trichilia (familia Meliaceae) ha despertado interés en la Flora Brasileña, debido a las actividades biológicas de sus metabolitos secundarios. Este género tiene especies distribuidas por toda América tropical, reconocidas por su gran importancia económica y alto valor comercial. Su composición está formada principalmente por terpenoides (triterpenos, sesquiterpenos, limonoides y esteroides) y polifenoles (flavonoides y taninos). En la medicina popular, este género se utiliza en preparaciones como antimicrobianos, antimaláricos, antivirales y antioxidantes. Estudios realizados con varias especies del género Trichilia muestran que estas plantas tienen actividad antioxidante, anticolinesterasa, antimicrobiana frente a patógenos de gran importancia clínica, neuroprotectora, antiinflamatoria y antiinflamatoria. Además, las actividades analgésicas, antineoplásicas, hepatoprotectoras e inmunomoduladoras también se han investigado y probado en la literatura. Con base en el gran potencial biotecnológico del género Trichilia, a partir de los escasos estudios sobre sus aplicaciones en salud y en vista de la importancia de la búsqueda de sustancias naturales que tengan actividades terapéuticas efectivas, esta revisión destaca las principales aplicaciones terapéuticas de las especies de este género encontrado. en la literatura, y para estimular nuevos estudios sobre el uso de estas plantas en el tratamiento de varias enfermedades. Para ello, se realizó una encuesta sobre el tema en las principales fuentes de investigación científica en el período 2005 a 2020, llevándonos a concluir que este género puede ser un poderoso aliado en la búsqueda de nuevas medicinas a base de plantas.
\end{abstract}

Palabras clave: Género Trichilia; Familia Meliaceae; Tratamiento; Enfermedades; Plantas medicinales.

\title{
1. Introduction
}

Plants are considered raw materials for the development of new drugs, being an unlimited source of potentially active substances and many of them are used to help promote the treatment of numerous diseases that affect humans and other animals, as well as in the control of plant pests. Phytotherapy and the use of medicinal plants are ancient practices, constituting a set of knowledge from different users and practitioners, especially through oral or epicutaneous use. Approximately $60 \%$ of the world's population uses almost entirely plants for medication and herbal medicines have been recognized as an important source of therapeutically effective medicines (Hayouni et al., 2011; Schenkel, 1995). Medicinal plants are an important component of health care for the majority of the world's population: they constitute the main medical care for 70 to $95 \%$ of citizens in most developing countries and are increasingly used by a large number of people residing in wealthier countries (Eldin \& Dunford, 2001; Applequist et al., 2020).

Despite the widespread use of medicinal plants for therapeutic purposes, the possible existence of toxic effects is little known. Also, many of these plants have few studies validating their therapeutic action. Although popular knowledge has significantly contributed to the understanding of the effects of medicinal plants, the active principles, mechanism of action and toxicity of many of them are still poorly understood, thus justifying further study to scientifically validate the effectiveness of its constituents. Medicinal plants are important both for being suppliers of raw materials for drug synthesis, as well as for being used as therapeutic agents (Estevão et al., 2013; David, Wolfender \& Dias, 2014).

Plants of the Meliaceae family are chemically characterized by the presence of tetranortriterpenoids known as limonoids or meliacins (Silva, Gottlieb \& Dreyer, 1984). Of all plant families, Meliaceae is among the most useful for man, mainly for its high-quality woods and the ease with which some species can be grown in plantations (Pennington \& Styles, 1975). Besides, the Meliaceae family attracts great interest among phytochemicals interested in bioproducts due to its very complex chemical structures and biological activity.

In Brazil, eight genera belonging to the Meliaceae family have been reported namely Azadirachta, Carpa, Cedrela, Guarea, Melia, Swietenia, Toona and Trichilia (Pennington, Styles \& Taylor, 1981; Stefano, Calazans \& Sakuragui, 2014). The genus Trichilia was described by Browne in 1756, comprising 70 species distributed in Tropical America, Africa and the Indo-Malay region, of which approximately 53 species occur in Brazil. This genus has aroused interest among the Brazilian flora, due to the biological activities of secondary metabolites present in these plants (Pennington, Styles \& Taylor, 1981).

The main characters that define the genus Trichilia are related to the floral structure and the fruits. Flowers with stamens partially or completely fused carrying the anthers at the apex of the filaments or on the edge of the stem tube. The fruit 
is a loculicidal capsule, 2-3 (-4) valves, smooth to warty, sepals persistent or not in the fruits and locules 1-2 seeds (Pennington, 2016). This genus has the largest number of species in the Meliaceae family (Pennington, Styles \& Taylor, 1981). Phytochemical studies have revealed that it is rich in terpenoids (triterpenes, limonoids and steroids) and polyphenols (flavonoids and tannins) (Ramírez et al., 2000; Terra et al., 2009).

Therefore, based on the great biotechnological potential of the genus Trichilia, because of the scarce studies on its applications in health and on the importance of the search for natural substances that have effective therapeutic activities, this review is necessary, to stimulate investments in studies that seek in these plants a path for the production of new phytotherapeutic formulations for the pharmaceutical market.

\section{Methodology}

A survey was carried out on the theme between the years 2005 and 2020, in this period the largest number and the most relevant publications on the theme were found. The main sources of scientific research were consulted, using the DeCS (Health Science Descriptors): Meliaceae family; genus Trichilia, treatment, disease, antimicrobial, antifungal, antibacterial and antineoplasic, as well as its synonyms and correspondents in portuguese: família Meliaceae, gênero Trichilia, tratamento, doenças, antimicrobiano, antifúngico, antibacteriano and antineoplásico. The main sources of research used were: PubMed, Scielo, Thieme, EBSCO Host, Journals Capes and Flora do Brasil-JBRJ (Board1).

The analysis was divided into three stages. The first stage consisted of reading the title of the articles, where only those with related terms such as genus Trichilia were selected. Then, the second step was taken, in which the Summary or Abstract of the articles included in the first phase was read, among which those that mentioned some type of effective treatment for diseases using plants of the genus Trichilia were chosen. Finally, in the third and last stage, the full text of the articles selected in the second stage was evaluated, to elect articles that discussed the use and importance of species of the genus Trichilia used in the treatment of diseases and in combating pathogens that cause diseases in humans. Articles that mentioned only the empirical use of plants were excluded, in addition to studies carried out before 2005. It should also be noted that articles repeated in the databases and articles with incomplete research information were eliminated. We finished the work with 42 articles. Of these, we used 30 articles in our results, as they evaluated the therapeutic activities of the genus. The rest were used in our introduction and/or discussion. 
Board 1. Result of the survey carried out on the topic in the main research sources.

\begin{tabular}{|l|l|l|l|l|}
\hline Database & $\begin{array}{l}\text { Total } \\
\text { articles }\end{array}$ & $\begin{array}{l}\text { Excluded } \\
\text { after reading } \\
\text { the abstract }\end{array}$ & Read in full & Selected \\
\hline SCIELO & 20 & 19 & 1 & 1 \\
\hline LILACS & 10 & 7 & 3 & 3 \\
\hline PUBMED & 30 & 6 & 24 & 24 \\
\hline $\begin{array}{l}\text { CAPES } \\
\text { JOURNALS }\end{array}$ & 20 & 11 & 9 & 9 \\
\hline THIEME & 6 & 4 & 2 & 2 \\
\hline $\begin{array}{l}\text { FLORA } \\
\text { BRAZIL OF }\end{array}$ & 9 & 6 & 3 & 3 \\
\hline TOTAL & 75 & 53 & 42 & 42 \\
\hline
\end{tabular}

Databases used to search for articles: Scielo, LILACS, Pubmed, Capes Journals, Thieme and Flora of Brazil. Source: Authors (2021).

\section{Results and Discussion}

The genus Trichilia has a variety of species with proven therapeutic activities for the treatment of numerous diseases, being used to combat various pathogens that cause damage to human health. In this review, 11 species studied were found regarding their therapeutic activity. Figure 1 shows the species of the genus Trichilia and the number of articles found for each one.

Figure 1. Number of articles by Trichilia species.

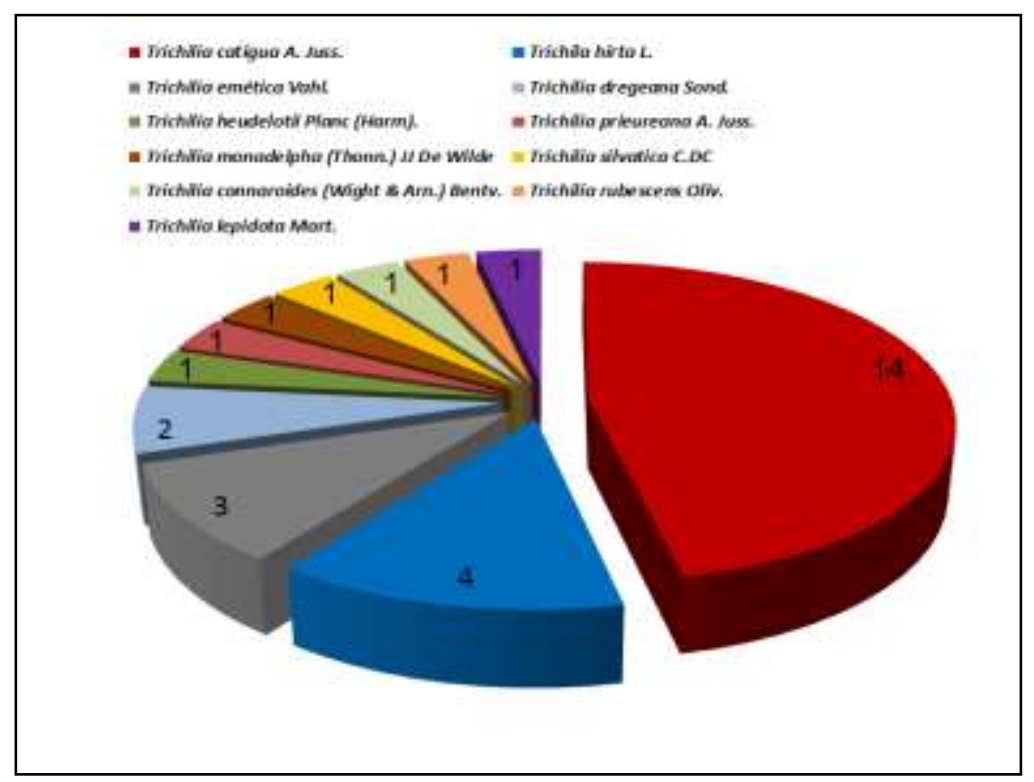

Number Articles found for each Trichilia species reporting its therapeutic activity, years 2005 to 2020. Source: Authors. 
Table 1 shows the number of articles published between 2005 and 2020 in Brazil and worldwide, highlighting the species of the genus Trichilia that were studied, the part of the plant used, as well as the form used in the tests, the therapeutic activity found and the constituents that may be responsible for this activity, totaling 30 articles. Importantly, of these, 15 articles were carried out in Brazil reaffirming the interest of Brazilian researchers in medicinal plants and their derivatives for the treatment and prevention of numerous diseases.

As for the preparation used in the tests, 16 articles were found using extracts, eight (09) articles used only the purified form (fractions) and, finally, four (05) articles tested both extracts and fractions, always highlighting the effectiveness of each one of them about the studied disease or pathogen.

Table 1. Survey of studies on the main therapeutic applications of the genus Trichilia.

\begin{tabular}{|c|c|c|c|c|c|c|}
\hline Species & Application & $\begin{array}{l}\text { Used } \\
\text { part }\end{array}$ & $\begin{array}{l}\text { Form } \\
\text { used }\end{array}$ & $\begin{array}{c}\text { Constituent of the } \\
\text { plant }\end{array}$ & Authors & $\begin{array}{c}\text { Year/ } \\
\text { Country }\end{array}$ \\
\hline $\begin{array}{c}\text { Trichilia } \\
\text { catigua A. } \\
\text { Juss. }\end{array}$ & antidepressant & bark & $\begin{array}{l}\text { Hydroalcoho- } \\
\text { lic extract }\end{array}$ & unreported & $\begin{array}{l}\text { Campos } \\
\text { et al. }\end{array}$ & $\begin{array}{l}\text { Brazil } \\
2005\end{array}$ \\
\hline $\begin{array}{c}\text { Trichilia } \\
\text { catigua A. } \\
\text { Juss. }\end{array}$ & Antinociceptive & bark & $\begin{array}{l}\text { Hydroalcoho- } \\
\text { lic extract }\end{array}$ & $\begin{array}{c}\text { flavonoid, } \\
\text { cinchonine IIB }\end{array}$ & $\begin{array}{l}\text { Viana } \\
\text { et al. }\end{array}$ & $\begin{array}{l}\text { Brazil } \\
2011\end{array}$ \\
\hline $\begin{array}{c}\text { Trichilia } \\
\text { catiguá A. } \\
\text { Juss. }\end{array}$ & Antioxidant & bark & $\begin{array}{l}\text { ethanolic } \\
\text { extract, ethyl } \\
\text { acetate } \\
\text { fraction, } \\
\text { butanolic } \\
\text { fraction }\end{array}$ & $\begin{array}{c}\text { phenolic } \\
\text { compounds, } \\
\text { flavonoids, } \\
\text { quercetin, rutin }\end{array}$ & $\begin{array}{l}\text { Kamdem } \\
\text { et al. }\end{array}$ & $\begin{array}{l}\text { Brazil } \\
2012\end{array}$ \\
\hline $\begin{array}{c}\text { Trichilia } \\
\text { catigua A. } \\
\text { Juss. }\end{array}$ & Antidepressant & bark & $\begin{array}{l}\text { ethyl acetate } \\
\text { fraction }\end{array}$ & phenylpropa-noid & Bonassoli et al. & $\begin{array}{c}\text { Brazil } \\
2012\end{array}$ \\
\hline $\begin{array}{c}\text { Trichilia } \\
\text { catigua A. } \\
\text { Juss. }\end{array}$ & neuroprotection & bark & $\begin{array}{l}\text { ethyl acetate } \\
\text { fraction }\end{array}$ & $\begin{array}{l}\text { epicaterquina, } \\
\text { cinchonina, } \\
\text { procianidina B2 }\end{array}$ & Truiti et al. & $\begin{array}{c}\text { Brazil } \\
2015\end{array}$ \\
\hline $\begin{array}{c}\text { Trichilia } \\
\text { catigua A. } \\
\text { Juss. }\end{array}$ & antiviral & bark & $\begin{array}{c}\text { Aqueous } \\
\text { acetone extract } \\
\text { Aqueous and } \\
\text { ethyl acetate } \\
\text { fraction }\end{array}$ & not reported & $\begin{array}{l}\text { Espada } \\
\text { et al. }\end{array}$ & $\begin{array}{l}\text { Brazil } \\
2015\end{array}$ \\
\hline $\begin{array}{c}\text { Trichilia } \\
\text { catigua A. } \\
\text { Juss. }\end{array}$ & antidiabetic & bark & $\begin{array}{l}\text { ethyl acetate } \\
\text { fraction }\end{array}$ & $\begin{array}{l}\text { procyanidin B2, } \\
\text { catechin, } \\
\text { chlorogenic acid, } \\
\text { cinchonine IIb IIa } \\
\text { Ia, epicatechin }\end{array}$ & $\begin{array}{l}\text { Gomes } \\
\text { et al. }\end{array}$ & $\begin{array}{l}\text { Brazil } \\
2017\end{array}$ \\
\hline $\begin{array}{c}\text { Trichilia } \\
\text { catigua A. } \\
\text { Juss. }\end{array}$ & $\begin{array}{c}\text { antioxidant, } \\
\text { anticholinesterase, } \\
\text { anti-fatigue }\end{array}$ & bark & $\begin{array}{l}\text { Hydroalcoho- } \\
\text { lic, aqueous, } \\
\text { chloroform, } \\
\text { hexane } \\
\text { extracts }\end{array}$ & $\begin{array}{l}\text { cinchonine, } \\
\text { procyanidins }\end{array}$ & $\begin{array}{l}\text { Martins } \\
\text { et al. }\end{array}$ & $\begin{array}{c}\text { Brazil } \\
2018\end{array}$ \\
\hline
\end{tabular}




\begin{tabular}{|c|c|c|c|c|c|c|}
\hline $\begin{array}{c}\text { Trichilia } \\
\text { catigua A. } \\
\text { Juss. }\end{array}$ & $\begin{array}{l}\text { antioxidant, anti- } \\
\text { inflammatory }\end{array}$ & bark & $\begin{array}{l}\text { ethyl acetate } \\
\text { fraction }\end{array}$ & $\begin{array}{l}\text { cinchonine, } \\
\text { chlorogenic } \\
\text { acid, } \\
\text { tannins, } \\
\text { epicatechin, } \\
\text { procyanidin }\end{array}$ & $\begin{array}{l}\text { Vicentini } \\
\text { et al. }\end{array}$ & $\begin{array}{c}\text { Brazil } \\
2018\end{array}$ \\
\hline $\begin{array}{c}\text { Trichilia } \\
\text { catigua A. } \\
\text { Juss. }\end{array}$ & $\begin{array}{l}\text { Antioxidant, } \\
\text { antiinflama-tory, } \\
\text { anti-amnesic }\end{array}$ & bark & $\begin{array}{l}\text { ethyl acetate } \\
\text { fraction }\end{array}$ & $\begin{array}{l}\text { epicatechins, } \\
\text { procyanidins } \\
\text { (B2) }\end{array}$ & $\begin{array}{l}\text { Godinho } \\
\text { et al. }\end{array}$ & $\begin{array}{l}\text { Brazil } \\
2018\end{array}$ \\
\hline $\begin{array}{c}\text { Trichilia } \\
\text { catigua A. } \\
\text { Juss. }\end{array}$ & $\begin{array}{l}\text { neuroactive, } \\
\text { neuroprotective }\end{array}$ & bark & $\begin{array}{l}\text { Aqueous } \\
\text { extract }\end{array}$ & $\begin{array}{c}\text { flavan-3-ol, } \\
\text { phenylpropa-noid }\end{array}$ & Bernardo et al. & $\begin{array}{c}\text { Portugal } \\
2018\end{array}$ \\
\hline $\begin{array}{c}\text { Trichilia } \\
\text { catigua A. } \\
\text { Juss. }\end{array}$ & $\begin{array}{l}\text { Antioxidant, anti- } \\
\text { inflammatory, }\end{array}$ & bark & $\begin{array}{l}\text { ethyl acetate } \\
\text { fraction }\end{array}$ & $\begin{array}{c}\text { epicatechins, } \\
\text { procyanidins (B2, } \\
\text { B4 e C1), } \\
\text { cinchonine (Ia,Ib, } \\
\text { IIa e IIb) }\end{array}$ & $\begin{array}{l}\text { Godinho } \\
\text { et al. }\end{array}$ & $\begin{array}{l}\text { Brazil } \\
2018\end{array}$ \\
\hline $\begin{array}{c}\text { Trichilia } \\
\text { catigua A. } \\
\text { Juss. }\end{array}$ & $\begin{array}{l}\text { antioxidant, } \\
\text { neuroprotection }\end{array}$ & bark & $\begin{array}{l}\text { ethyl acetate } \\
\text { fraction }\end{array}$ & flavonoids & $\begin{array}{l}\text { Panizzon } \\
\text { et al. }\end{array}$ & $\begin{array}{c}\text { Brazil } \\
2019\end{array}$ \\
\hline $\begin{array}{c}\text { Trichilia } \\
\text { catigua A. } \\
\text { Juss. }\end{array}$ & $\begin{array}{l}\text { Antimicrobial: fungi } \\
\text { and bacteria }\end{array}$ & bark & $\begin{array}{l}\text { Aqueous } \\
\text { acetone } \\
\text { extract, } \\
\text { aqueous and } \\
\text { ethyl acetate } \\
\text { fraction }\end{array}$ & $\begin{array}{l}\text { chlorogenic } \\
\text { acids, flavan-3- } \\
\text { ols, } \\
\text { proanthocyanid } \\
\text { ins, citric acid, } \\
\text { catechin-3-O-a- } \\
\text { L-ramhamide- } \\
\text { epicatechin }\end{array}$ & $\begin{array}{l}\text { Ritter } \\
\text { et al. }\end{array}$ & $\begin{array}{l}\text { Brazil } \\
2019\end{array}$ \\
\hline $\begin{array}{c}\text { Trichilia } \\
\text { silvatica } \\
(\text { C.DC })\end{array}$ & $\begin{array}{l}\text { Anti-inflammatory, } \\
\text { antioxidant, } \\
\text { antiproliferative }\end{array}$ & leaf, bark & $\begin{array}{l}\text { Methanolic } \\
\text { extract }\end{array}$ & $\begin{array}{l}\text { phenolic } \\
\text { compounds, } \\
\text { flavonoids, } \\
\text { flavonol and } \\
\text { tannins }\end{array}$ & $\begin{array}{l}\text { Da Silva } \\
\text { et al. }\end{array}$ & $\begin{array}{l}\text { Brazil } \\
2018\end{array}$ \\
\hline $\begin{array}{l}\text { Trichilia } \\
\text { dregeana }\end{array}$ & $\begin{array}{l}\text { Anti-inflammatory, } \\
\text { anticholinesterase }\end{array}$ & leaf & $\begin{array}{l}\text { Ethyl acetate, } \\
\text { ethanolic and } \\
\text { aqueous } \\
\text { extract }\end{array}$ & not reported & $\begin{array}{l}\text { Eldeen } \\
\text { et al. }\end{array}$ & $\begin{array}{l}\text { South } \\
\text { Africa } \\
2005\end{array}$ \\
\hline $\begin{array}{c}\text { Trichilia } \\
\text { emetica } \\
\text { Vahl. }\end{array}$ & $\begin{array}{c}\text { Hepatoprotective, } \\
\text { anti-bacterial }\end{array}$ & root & $\begin{array}{l}\text { Aqueous } \\
\text { extract, } \\
\text { Ethyl ether } \\
\text { fraction }\end{array}$ & $\begin{array}{l}\text { limonoids, } \\
\text { polyphenols }\end{array}$ & $\begin{array}{l}\text { Germano } \\
\text { et al. }\end{array}$ & $\begin{array}{l}\text { Africa } \\
2005\end{array}$ \\
\hline $\begin{array}{c}\text { Trichila } \\
\text { emética } \\
\text { Vahl. }\end{array}$ & Antimicrobial & fruit & $\begin{array}{l}\text { Methanolic } \\
\text { extract }\end{array}$ & $\begin{array}{c}\text { tannins, } \\
\text { polyphenols, sterols, } \\
\text { triterpenes }\end{array}$ & $\begin{array}{l}\text { Geyd } \\
\text { et al. }\end{array}$ & $\begin{array}{c}\text { Ethiopia } \\
2005\end{array}$ \\
\hline $\begin{array}{c}\text { Trichilia } \\
\text { emética Vahl. }\end{array}$ & $\begin{array}{l}\text { Antidiarrheal, } \\
\text { antimicrobial }\end{array}$ & leaf & $\begin{array}{l}\text { ethyl acetate } \\
\text { fraction }\end{array}$ & $\begin{array}{l}\text { polyphenols, } \\
\text { flavonoids }\end{array}$ & $\begin{array}{l}\text { Konaté } \\
\text { et al. }\end{array}$ & $\begin{array}{l}\text { Africa } \\
2015\end{array}$ \\
\hline $\begin{array}{l}\text { Trichilia hirta } \\
\text { L. }\end{array}$ & $\begin{array}{l}\text { Immunostimula-tor, } \\
\text { cytotoxic cancer } \\
\text { cells }\end{array}$ & root & $\begin{array}{l}\text { Aqueous, } \\
\text { extract }\end{array}$ & $\begin{array}{l}\text { saponins, } \\
\text { tannins, lipids, } \\
\text { glycosides, } \\
\text { flavonoids, }\end{array}$ & $\begin{array}{l}\text { Sosa } \\
\text { et al. }\end{array}$ & $\begin{array}{l}\text { Cuba } \\
2010\end{array}$ \\
\hline
\end{tabular}




\begin{tabular}{|c|c|c|c|c|c|c|}
\hline & & & & $\begin{array}{l}\text { carbohydra-tes, } \\
\text { coumarins }\end{array}$ & & \\
\hline $\begin{array}{c}\text { Trichilia hirta } \\
\text { L. }\end{array}$ & Immunostimula-tor & root & Ethanol extract & $\begin{array}{l}\text { saponins, } \\
\text { coumarins, } \\
\text { polyphenols, } \\
\text { tannins, flavonoids, } \\
\text { triterpenes, } \\
\text { alkaloids, quinoins, } \\
\text { glycosides }\end{array}$ & $\begin{array}{l}\text { Sosa } \\
\text { et al. }\end{array}$ & $\begin{array}{l}\text { Cuba } \\
2011\end{array}$ \\
\hline $\begin{array}{c}\text { Trichilia hirta } \\
\text { L. }\end{array}$ & antitumor & leaf & $\begin{array}{l}\text { Aqueous } \\
\text { extract }\end{array}$ & $\begin{array}{l}\text { triterpenes, steroids, } \\
\text { saponins, } \\
\text { coumarins, reducing } \\
\text { sugars, phenols, } \\
\text { tannins, flavonoids, } \\
\text { carbohydrates/glyco } \\
\text { sides }\end{array}$ & $\begin{array}{l}\text { Sosa } \\
\text { et al. }\end{array}$ & $\begin{array}{l}\text { Cuba } \\
2013\end{array}$ \\
\hline $\begin{array}{l}\text { Trichilia } \\
\text { hirta L. }\end{array}$ & Anticancer & leaf & $\begin{array}{l}\text { Polysacchari } \\
\text { de fraction }\end{array}$ & $\begin{array}{l}\text { flavonoids, } \\
\text { limonoids, tannins }\end{array}$ & $\begin{array}{l}\text { Hernández et } \\
\text { al. }\end{array}$ & $\begin{array}{l}\text { Cuba } \\
2013\end{array}$ \\
\hline $\begin{array}{l}\text { Trichilia } \\
\text { rubescens }\end{array}$ & Antimalarial & leaf & $\begin{array}{l}\text { methanolic, } \\
\text { ethyl acetate } \\
\text { extracts }\end{array}$ & $\begin{array}{l}\text { limonoids, } \\
\text { trichirubins }\end{array}$ & $\begin{array}{l}\text { Krief } \\
\text { et al. }\end{array}$ & $\begin{array}{c}\text { Uganda } \\
2006\end{array}$ \\
\hline $\begin{array}{l}\text { Trichilia } \\
\text { prieureana } \\
\text { A. Juss. }\end{array}$ & Antimicrobial & bark & ethanol extract & Not reported & $\begin{array}{l}\text { Kuglerova } \\
\text { et al. }\end{array}$ & $\begin{array}{l}\text { Africa } \\
2007\end{array}$ \\
\hline $\begin{array}{c}\text { Trichilia } \\
\text { drageana }\end{array}$ & Antimicrobial & leaf & $\begin{array}{l}\text { aqueous, } \\
\text { organic } \\
\text { extracts }\end{array}$ & Not reported & $\begin{array}{l}\text { Naidoo } \\
\text { et al. }\end{array}$ & $\begin{array}{c}\text { South } \\
\text { Africa } \\
2013\end{array}$ \\
\hline $\begin{array}{c}\text { Trichilia } \\
\text { heudelotii } \\
\text { Planc (Harm) }\end{array}$ & $\begin{array}{l}\text { antifungal, } \\
\text { cytotoxic }\end{array}$ & $\begin{array}{l}\text { leaf, } \\
\text { bark }\end{array}$ & $\begin{array}{l}\text { aqueous, } \\
\text { acetone, etanol } \\
\text { extracts }\end{array}$ & $\begin{array}{l}\text { steroids, } \\
\text { chalcones, } \\
\text { alkaloids, } \\
\text { tannins, phenols, } \\
\text { anthraquinone, } \\
\text { glycosides, } \\
\text { flavonoids }\end{array}$ & $\begin{array}{l}\text { Opawale } \\
\text { et al. }\end{array}$ & $\begin{array}{c}\text { Nigeria } \\
2015\end{array}$ \\
\hline $\begin{array}{c}\text { TRICHILIA } \\
\text { CONNAROI } \\
\text { DES } \\
\text { (WIGHT \& } \\
\text { ARN.) } \\
\text { BENTV. }\end{array}$ & anti-hyperlipidemic & leaf & $\begin{array}{l}\text { methanolic, } \\
\text { chloroform } \\
\text { extracts }\end{array}$ & $\begin{array}{l}\text { tetranorterpeno } \\
\text { ides, triterpenes }\end{array}$ & $\begin{array}{l}\text { Subarrao \& } \\
\text { Ashok }\end{array}$ & $\begin{array}{l}\text { India } \\
2011\end{array}$ \\
\hline $\begin{array}{l}\text { Trichilia } \\
\text { monadelpha } \\
\text { (Thonn.) JJ } \\
\text { De Wilde }\end{array}$ & $\begin{array}{l}\text { anti- } \\
\text { anaphylactics }\end{array}$ & bark & $\begin{array}{l}\text { ethanolic, } \\
\text { petroleum } \\
\text { ether extracts }\end{array}$ & $\begin{array}{l}\text { alkaloids, } \\
\text { flavonoids }\end{array}$ & $\begin{array}{l}\text { Ben } \\
\text { et al. }\end{array}$ & $\begin{array}{l}\text { Ghana } \\
2016\end{array}$ \\
\hline $\begin{array}{l}\text { Trichilia } \\
\text { lepidota }\end{array}$ & $\begin{array}{l}\text { cytotoxicity against } \\
\text { leukemic cells }\end{array}$ & $\begin{array}{l}\text { leaf, } \\
\text { fruit }\end{array}$ & $\begin{array}{l}\text { hexanic, } \\
\text { methanolic } \\
\text { extracts, } \\
\text { fractions of } \\
\text { limonoids }\end{array}$ & $\begin{array}{c}\text { PROTOLIMONOID } \\
\mathrm{S}\end{array}$ & $\begin{array}{l}\text { Terra } \\
\text { et al. }\end{array}$ & $\begin{array}{c}\text { Brazil } \\
2013\end{array}$ \\
\hline
\end{tabular}

Articles published from 2005 to 2020 in Brazil and the world, standing out as a species of the genus Trichilia that were studied, the part of the plant used, as well as the form used, the therapeutic application found, and the constituents that may be responsible for this activity. Source: Authors. 
In the phytochemical analysis, many of the Trichilia species revealed the presence of several compounds such as flavonoids, cinchonines, catechins, epicatechins and procyanidins linked to the antioxidant, anti-inflammatory, anti-amnesic and antiproliferative action. The presence of limonoids, protolimonoids and triterpenes linked to the antimicrobial and cytotoxic action against tumor cells was also observed, in addition to the presence of tannins linked to the antimicrobial action. These findings prove the uses of these species in traditional medicine. In Board 2 we report the popular name of the species of the genus Trichilia found in this review of the popular name and its traditional use.

Board 2. Popular name and use in the traditional medicine of Trichilia species mentioned in this review.

\begin{tabular}{|c|c|c|}
\hline Species & Popular name & Traditional use \\
\hline $\begin{array}{l}\text { Trichilia catigua A. } \\
\text { Juss. }\end{array}$ & catuaba, big catuaba & $\begin{array}{l}\text { fatigue, stress, impotence, memory } \\
\text { deficit }\end{array}$ \\
\hline Trichila hirta $\mathbf{L}$. & $\begin{array}{l}\text { cedrinho, Jubabán, catiguá-arco-de- } \\
\text { peneira, cedrillo, cedrillo colorado, red } \\
\text { cedro, trompillo }\end{array}$ & cancer, ulcer, asthma \\
\hline Trichilia emética Vahl. & mafurreira, christmas Mahogany & $\begin{array}{l}\text { abdominal pain, dermatitis, } \\
\text { hemorrhoids, jaundice, chest pain, } \\
\text { diuretic, emetic, purgative, labor } \\
\text { induction }\end{array}$ \\
\hline $\begin{array}{l}\text { Trichilia dregeana } \\
\text { Sond. }\end{array}$ & $\begin{array}{l}\text { forest mahogany, forest christmas } \\
\text { Mahogany, cape mahogany, thunder } \\
\text { tree, Christmas bells, red ash }\end{array}$ & $\begin{array}{l}\text { Hepatitis, stomach problems, back } \\
\text { pain, kidney pain, syphilis, purging, } \\
\text { leprosy, insomnia }\end{array}$ \\
\hline $\begin{array}{l}\text { Trichilia } \\
\text { heudelotii Planc } \\
\text { (Harm). }\end{array}$ & Akoko rere & $\begin{array}{l}\text { microbial infections (gastrointestinal } \\
\text { infections, gonorrhea) }\end{array}$ \\
\hline $\begin{array}{c}\text { Trichilia prieureana A. } \\
\text { Juss. }\end{array}$ & monkey apple, mtimaji, lomaran & $\begin{array}{c}\text { rheumatism, arthritis, bronchitis, } \\
\text { tetanus, malaria, ascariasis, purging, } \\
\text { fever, cough, constipation, poisoning, } \\
\text { ascites }\end{array}$ \\
\hline $\begin{array}{l}\text { Trichilia monadelpha } \\
\text { (Thonn.) JJ De Wilde }\end{array}$ & otanduro (Twi), tenuba (Nzema) & $\begin{array}{l}\text { allergic or anaphylactic reactions, } \\
\text { inflammatory disorders (arthritis), } \\
\text { epilepsy, depression, pain, psychosis }\end{array}$ \\
\hline $\begin{array}{l}\text { Trichilia silvatica } \\
\text { C.DC }\end{array}$ & White-rose, white catiguá, red agouti & rheumatism (arthritis) \\
\hline $\begin{array}{c}\text { TRICHILIA } \\
\text { CONNAROIDES } \\
\text { (WIGHT \& ARN.) } \\
\text { BENTV. }\end{array}$ & $\begin{array}{c}\text { Karai ,karaivilangu (Tamil), korakudi, } \\
\text { kuravatti (malaiala), kora } \\
\text { (Kannada) }\end{array}$ & $\begin{array}{l}\text { cholera, arthritis, pharyngitis, } \\
\text { tonsillitis }\end{array}$ \\
\hline $\begin{array}{c}\text { Trichilia rubescens } \\
\text { Oliv. }\end{array}$ & - & malaria \\
\hline $\begin{array}{l}\text { Trichilia lepidota } \\
\text { Mart. }\end{array}$ & cedrinho & - \\
\hline
\end{tabular}

Popular name and use in the traditional medicine of the species of the genus Trichilia. (-) not found. Source: Authors.

The main therapeutic actions found for each species were reported below:

\section{Trichilia catigua A. Juss}

This species was the most studied regarding its therapeutic action. Perhaps this fact can be justified by its wide distribution, which can be found in several countries in South America (Godinho et al., 2018). It is worth mentioning that most studies involving this species were carried out in Brazil, which can be justified by being a species native to the Brazilian Flora. 
Gomes et al. (2017), evaluated the antidiabetic activity of the Trichlia catigua ethyl acetate fraction in type I diabetic rats and reported that this fraction improved biochemical parameters, such as hyperglycemia, alkaline phosphatase, alanine and aspartate aminotransferase. An improvement in glucose homeostasis and pancreatic morphology was observed, in addition to the inhibition in the development of diabetic nephropathy in diabetic rats, resulting in a reduction in renal tissue damage by reducing fibrosis. Do Nascimento et al. (2019) also evaluated the effects of the ethyl acetate fraction of this plant in type I diabetic rats, but investigated the neuroprotective role of this fraction, in the total population of enteric neurons in the jejunum of these rats. As a result, it was observed that Trichlia catigua A. Juss provided partial protection against diabetic neuropathy in the enteric nervous system. These works, together, showed that the fraction of Trichlia catigua A. Juss is an option that can be used in therapeutic formulations to improve many problems resulting from diabetes.

We found studies evaluating the antioxidant action of Trichilia catigua, using different tests and different models such as Kandem et al. (2012), who observed the antioxidant activity of Trichilia catigua extracts and fractions in different concentrations (10-400ug/mL), using models such as elimination of DPPH (IC50) and lipid peroxidation induced by Fe2+, and attributed this action to a flavonoid (quercetin). After many years, Vicentini et al. (2018), evaluated the effect of the ethyl acetate fraction of Trichilia catigua $(200 \mathrm{mg} / \mathrm{kg})$ in the model of colitis induced by $2,4,6$-trinitrobenzene sulfonic acid (TNBS) in rats. Treatment with the extract of this plant prevented oxidative stress, reduced inflammation in seven days and improved tissue recovery. Confirming the antioxidant action of this plant, the work of Godinho et al. (2018) studied the ethyl acetate fraction of Trichilia catigua, but in a reperfusion ischemia model. This fraction showed antioxidant activity (reducing oxidative stress) and has anti-inflammatory action (reducing the activity of glial cells and myeloperoxidase) in the initial phase of reperfusion ischemia, contributing to the long-term antiamnesic effect. In this same line of research, in another work in the same year, Godinho et al. demonstrated that Trichilia catigua has antioxidant and anti-inflammatory activities, significantly decreasing the levels of (myeloperoxidase and carbonylation protein) in the transient global cerebral ischemia in rats, which also contributed to the protective effect of memory. The study by Martins et al. (2018) corroborates these findings and brings new data regarding the therapeutic actions of this plant showing that the extract of Trichilia catigua not only has antioxidant activities for the elimination of DPPH (EC50 $43 \mathrm{ug} / \mathrm{mL}$ ) but also anticholinesterases. , in addition to exerting a slight protective effect on forced exercise and mouse fatigue. All of these studies demonstrate that this plant has an antioxidant action and can be used in herbal medicines for this purpose, in addition to being promising in combating neurodegenerative diseases. It is known in the literature and the works presented here also reported that flavonoids (quercetin, procyanidin, epicatechin and cinchonine) have antioxidant and anti-inflammatory effects and Trichilia catigua is rich in flavonoids.

This plant was evaluated for its protective effect against neurodegenerative processes. In the work of Truiti et al. (2015), the neuroprotective effects of a fraction of ethyl acetate from Trichilia catigua were evaluated in mice submitted to an in vivo model of cerebral ischemia. It was observed that the spatial cognitive deficits were neutralized by the administration of Trichilia catigua extract in concentrations (200, 400 and $800 \mathrm{ug} / \mathrm{kg}$ ). This extract significantly increased the number of intact cells in the hippocampus in mice. The extract of this plant promoted functional recovery, decreased the delayed loss of cells of the hippocampus, revealing its neuroprotective action. Bernardo et al. (2018), investigated the action of the aqueous extract of the bark of this plant as a double inhibitor of monoamine oxidase A (MAO-A), acetylcholinesterase (AChE) and explored its antioxidant potential through interaction with the xanthine/xanthine oxidase pathway, observed that this extract inhibited A (MAO-A) with a dose-dependent effect. Besides, the extract was shown to be an antioxidant by inhibiting the xanthine/xanthine oxidase pathway. Trichilia catigua extract demonstrated neuroactive and neuroprotective potential in the range of non-toxic concentrations $(0.063-500 \mu \mathrm{g} / \mathrm{mL})$ in SH-SY5Y cells of human neurons. According to Eldeen, Elgorashi and Van Staden (2005) traditionally, medicinal plants have been used successfully to improve cognitive function and to relieve other symptoms associated with Alzheimer's disease. 
Trichilia catigua was also analyzed for possible antidepressant effects in rodents, as well as the possible mechanisms involved in their actions. Oral treatment with Trichilia catigua extract (200ug / $\mathrm{kg}$ and 400ug/kg) produced effects of the antidepressant type in the forced swimming model, decreasing the immobility time of mice and rats. It was found that this action would be linked to the action of the extract in modulating the dopaminergic pathway (Campos et al., 2005). The study by Taciany Bonassoli et al. (2012) also demonstrated the antidepressant effect of acute administration of the ethyl acetate fraction of Trichilia catigua in mice, using the same forced swim test and included the forced suspension test. It was observed that the subchronic administration of Trichilia catigua $(400 \mathrm{ug} / \mathrm{kg})$ promoted effects of the antidepressant type in mice, observed by the decrease in the immobility time in the tests. Neural proliferation was also observed within 24 hours after fraction administration, which did not influence neuron survival at 7 and 15 days after treatment. New therapeutic options are necessary with antidepressant action, due to the lack of effect of current medications in some patients and due to the large number of side effects exhibited by these (Campos et al., 2005). Trichilia catigua as an antidepressant and may be a promising alternative for herbal formulations in this regard.

The study by Espada et al. (2015) sought to evaluate the antiviral activity of the crude extract and the aqueous and ethyl acetate fractions obtained from Trichilia catigua in the replication of the Herpes simplex virus (HSV-1), bovine herpesvirus (BoHV-1) and poliovirus (PV-1). Both the crude extract and the fractions showed low toxicity (CC50> $400 \mathrm{ug} / \mathrm{ml})$ and high antiviral effect for all viruses, with inhibitory concentration (IC50 2.44-34.25 ug/ml) for herpesvirus and (IC50 0,67$1.8 \mu \mathrm{g} / \mathrm{ml}$ ) for PV-1. Ritter et al. (2019), on the other hand, carried out work with crude extract from the bark of Trichlia catigua A. Juss and its fractions of ethyl and aqueous acetate. Its objective was to analyze the antimicrobial action against bacteria and fungi. Again this plant showed excellent results. Both the extract and the fraction presented (CIM $500 \mathrm{ug} / \mathrm{mL}$ ) for Bacillus subtilis. For Staphylococcus aureus, Pseudomonas aeruginosa and Escherichia coli (CIM>1000 ug/mL) for all tested compounds. Concerning yeasts, the crude extract showed (CIM $250 \mathrm{ug} / \mathrm{mL}$ ) for Candida tropicalis and Candida parapsilosis. However, the most significant result was for Candida albicans (CIM $125 \mathrm{ug} / \mathrm{mL}$ ) of the ethyl acetate fraction. For Enterococcus faecium considered resistant to current drugs, the crude extract and fraction of ethyl acetate revealed excellent results with (CIM $156.5 \mathrm{ug} / \mathrm{mL}$ ). Thus, it was demonstrated that the extract and fractions of Trichilia catigua are also promising for future studies, aiming at antiviral, antibacterial and antifungal drugs. The antimicrobial action of the tannins present in this plant is already known.

Evaluating antinociceptive activity, Viana et al. (2011) carried out a study with the hydroalcoholic extract in behavioral models of mice nociception, where they observed that this extract has antinociceptive effects. According to the authors, this effect is mainly associated with the activation of the dopaminergic system and, to a lesser extent, with the interaction with the opioid pathway.

The work involving Trichilia catigua showed that this plant is a promising candidate for new herbal formulations, with antimicrobial, antidepressant, neuroactive, analgesic and antioxidant action. Trichilia catigua showed interaction with the dopaminergic pathway, being also an inhibitor of the monoamine oxidase pathway and the acetylcholinesterase pathway. It is important to note that its extracts and fractions did not prove to be toxic until high concentrations.

\section{Trichilia hirta L.}

Trichilia hirta L. was the second most studied species, mainly to prove its popular antineoplastic and immunostimulatory use. Sosa et al. (2010), evaluated the immunorestorative and cytotoxic activity of aqueous extracts of Trichilia hirta L. root. The administration of this extract increased the global and differential leukocyte count in immunosuppressed mice, the weight of the thymus, as well as the cellularity of the bone marrow. The aqueous extract showed selective cytotoxicity against T-47D and SK-mel-3 tumor cells compared to the non-tumor strain (VERO). The results indicate 
that Trichilia hirta $\mathrm{L}$. has a significant in vivo immunorestorative effect and selective cytotoxicity, and may be a promising alternative to cancer therapy (Sosa et al., 2010). In another study by Sosa et al. (2011), with the ethanolic extract obtained from the roots of Trichlia hirta L., they evaluated the leukocyte stimulating effect of these extracts in BALB/C mice. The application of $976 \mathrm{ug} / \mathrm{kg}$ of the extract increased the leukocyte count by 15 to $33 \%$. Also, a dose of $82 \mathrm{ug} / \mathrm{kg}$ significantly increased the total number of leukocytes with 4 days of study $(\mathrm{P}<0.05)$. The results indicated that Trichlia hirta L. exhibits leukocyte-stimulating activity and makes it a promising alternative for the development of an immunoprotective agent (Sosa et al., 2011).

Studies by Hernandez et al. (2013) showed that the polysaccharide-rich fraction of Trichilia hirta L. showed antiproliferative activity against lung cancer cells and cells of the human cervical carcinoma, whereas control cells (fibroblasts) did not have their affected proliferation. Sosa, Mora Gonzalez, Morris and Humberto (2013) also reported that Trichilia hirta L. leaf extract showed selective cytotoxicity for breast cancer and melanoma cells compared to non-tumor cells (VERO) (Sosa, Mora Gonzalez, Morris \& Humberto, 2013) These results confirm studies that showed the selective cytotoxic action of the extract for cancer cells.

These studies point to strong evidence and provide a basis for the popular use of this plant, but more research is needed on its neoplastic potential. The presence of saponins, tannins, flavonoids, carbohydrates and coumarins was observed in these extracts. Flavonoids are already known to have an immunomodulatory action. The studies cited here to pave the way for researchers interested in studies on new therapies to fight cancer.

\section{Trichilia emetica Vahl.}

We find three articles about this species. One of these studies assesses the antibacterial and hepatoprotective action of the aqueous extract and of the ethyl ether fractions of the Trichilia emetica Vahl root, the hepatoprotective effect was significant $(\mathrm{P}<0.05)$ at the dose of $1000 \mu \mathrm{g} / \mathrm{ml}$, both on the plasma membrane and mitochondrial function. Extract and fraction showed no toxicity even at very high concentrations (LC50>1000 $\mu \mathrm{g} / \mathrm{mL}$ ). The fraction showed better activity against Streptococcus pneumoniae and Moraxella catarrhalis (CIM 7.80 and $125.00 \mu \mathrm{g} / \mathrm{mL}$ ), Staphylococcus aureus and Streptococcus pyogenes (CIM 15.60 and $62.50 \mu \mathrm{g} / \mathrm{mL}$ ) and Haemophilus influenzae (CIM $125 \mu \mathrm{g} / \mathrm{mL}$ ). The extract was only effective against the strains tested in concentrations above (CIM 500 $\mu \mathrm{g} / \mathrm{mL})($ Germano et al., 2005). In the same year, another study also evaluated the antimicrobial activity of the methanolic extract of the fruit of this plant, where inhibition of the growth of five strains of fungi of great medical importance (Candida albicans, Cryptococcus neoformans, Aspergillus flavus, Tricophyton mentagrophytes and Tricophyton violacium) at concentrations of 400, 500 and $1000 \mu \mathrm{g} / \mathrm{mL}$, but against bacteria, no significant efficacy was observed (Geyid et al., 2005).

Corroborating these studies, the work of Konaté, Yomalan, Sytar and Brestic (2015) showed that the fraction of ethyl acetate Trichilia emetica Vahl. has antibacterial activity against strains of Gram-negative and Gram-positive bacteria (Staphylococcus aureus, Escherichia coli, Pseudomonas aeruginosa, Salmonella typhi, Shigella boydii, Shigella flexneri and Shigella dysenteriae) CIM 156-1250ug/mL. In well diffusion tests, an inhibition zone (14-24mm) was found for all tested strains. Strains of bacteria were more sensitive to this fraction than ciprofloxacin. This fraction also had positive effects against diarrhea $(300 \mathrm{ug} / \mathrm{mL})$ induced by castor oil in mice. This result provided a scientific basis for the popular use of this plant for the treatment of gastroenteritis.

Infectious diseases are the leading cause of death in the world. The clinical effectiveness of many existing antibiotics is being threatened by the emergence of multidrug-resistant pathogens. Plant extracts are readily available for this area, due to their ability to produce compounds that confer an antimicrobial defense (Eldeen, Elgorashi \& Van Staden, 2005). The fractions of this plant have shown promise for future studies as antifungal and antibacterial, including in the fight against pathogens of 
great clinical importance. It is important to highlight that a protective action of hepatocytes against damage induced by CCL4 was observed. The studies cited here highlighted polyphenols and tannins as the possible responsible for the antimicrobial action of this plant and in no experiment was it toxic until high concentrations.

\section{Trichilia dregeana Sond.}

With this species, we found two studies. The first of 2005, evaluating the action of three extracts of Trichilia dregeana Sond. (ethanolic, aqueous and ethyl acetate) for antibacterial, anti-inflammatory (COX-1 and COX-2), mutagenic and anticholinesterase activities. The ethyl acetate leaf extract demonstrated selective inhibition of COX-2 (81\%) confirming its anti-inflammatory action. It was also observed that the ethyl acetate extract of the bark inhibited (55\%) the acetylcholinesterase pathway, confirming its anticholinesterase action. None of the extracts showed mutagenic activity and as for antibacterial activity, the ethanolic extract of the leaves against Escherichia coli (CIM 3.1ug/mL) stood out (Eldeen, Elgorashi \& Van Staden, 2005).

Subsequently, a study investigated the in vitro antimicrobial activity of this plant. It was observed that the aqueous and acetone extract showed activity against the pathogen Ureaplasma urealyticuma (CIM $0.25 \mathrm{ug} / \mathrm{mL}$ ). Besides, the combination of the aqueous extract of Trichilia dregeana Sond. with that of Albizia adianthifolia it showed excellent activity against Oligella ureolytica (MIC $0.75 \mathrm{ug} / \mathrm{mL}$ ) with high cell viability (IC50 $98 \mathrm{ug} / \mathrm{mL}$ ) for kidney epithelial cells (Naidoo, van Vuuren, van Zyl \& de Wet, 2013). None of the studies investigated the possible responsible for the mentioned activities, showing the need for further research in this regard.

\section{Trichilia heudelotii Planc (Harm).}

Only one study was found with this species where the antifungal and cytotoxic activities of the extracts (aqueous, acetone and ethanolic) of the bark, leaves and stems of Trichilia heudelotii Planc (Harm) were evaluated. As a result, it was observed that at the concentration of $50 \mathrm{ug} / \mathrm{mL}$, the most significant inhibition zone was the acetone extract from the leaves against Candida albicans $(17.00 \mathrm{~mm}$ ), followed by the acetone and ethanolic extracts from the stem bark against Trichophyton rubrum and Candida albicans $(16.00 \mathrm{~mm})$. The ethanolic extracts of the leaves showed the lowest activity for Trichophyton rubrum $(6.00 \mathrm{~mm})$. The minimum inhibitory concentration of all extracts against the pathogens tested varied between (CIM 2.5 and $200 \mu \mathrm{g} / \mathrm{mL}$ ). The cytotoxic activity of the extracts, assessed by the brine shrimp lethality bioassay method, revealed significant cytotoxic effects (LC50 9.01 to $256.17 \mathrm{ug} / \mathrm{mL}$ ) (Opawale, Oyetayo \& Agbaje, 2015). Despite the promising activity of this plant against some human pathogenic fungi, high levels of toxicity of the extracts have been observed, future tests can be done to better investigate these actions. It is worth mentioning that tests were not carried out to show the selectivity index.

\section{Trichilia prieureana A. Juss.}

With Trichilia prieureana A. Juss. only one study was found using the ethanol extract of the bark to evaluate its popular use as an antimicrobial. It was found that this extract strongly inhibited the tested Gram-positive bacteria (Enterococcus faecalis and Staphylococcus aureus) in very low concentration (CIM $0.25 \mathrm{ug} / \mathrm{mL}$ ) revealing that this plant is a promising antibacterial, giving scientific basis to its popular use (Kuglerova et al., 2007). We emphasize, in agreement with the authors, the need for further studies on this species regarding its antibacterial action. It is worth mentioning the need for studies evaluating the cytotoxic action of this plant. 


\section{Trichilia monadelpha (Thonn.) JJ De Wilde}

A study was found with ethanol extracts and petroleum ether from the stem bark of Trichilia monadelpha (Thonn.) JJ De Wilde, where their anti-anaphylactic and anti-inflammatory activities were evaluated, to prove their traditional use in the treatment of allergies and other inflammatory disorders. Both extracts were effective in decreasing mast cell degranulation and inhibiting TNF-alpha (TNF-a) to interleukin 6 (IL6) (100ug/kg). Also, the extracts also acted by preventing the destruction of the cell-matrix and tissue damage (100ug/kg) (Ben, Woode, Koffuor \& Asiamah, 2016). In the work it was reported that alkaloids are possibly responsible for the antianafilatic action of the ether extract and in the ethanolic extract flavonoids were found to have proven antiallergic action.

\section{Trichilia silvatica C.CD}

The research investigated the anti-inflammatory, antioxidant and antiproliferative activities of the methanolic extract of leaves and bark of Trichilia silvatica C.DC. As a result, it was observed that the extracts exhibited moderate antioxidant activity (IC50 $\leq 35.32 \mu \mathrm{g} / \mathrm{mL}$ ). Both extracts also inhibited carrageenan-induced paw edema after 2 and 4 hours. They also observed inhibition of leukocyte migration at 6 hours after administration of the extract. The methanolic extract of the leaves was particularly effective against the prostate cell line (EC50 $\leq 0.22 \mu \mathrm{g} / \mathrm{mL}$ ) (Da Silva et al., 2018). Unfortunately, this plant is being little explored regarding its therapeutic actions. We understand that Trichilia silvatica C.DC has a lot to offer, this is suggested by the presence of phenolic compounds, flavonoids and tannins found in its extracts.

\section{Trichilia connaroides (Wight \& Arn.) Bentv.}

Only one study evaluating the therapeutic property was found with Trichilia connaroides (Wight and Arn.) Bentv. Perhaps this fact can be explained, due to its more restricted geographical distribution, since most of it is found in India's rainforests. The purpose of the study was to investigate the anti-hyperlipidemic effect of chloroform and methanolic extracts from the leaves of Trichilia connaroides (Wight and Arn.) Bentv. in hypercholesterolemic rats and the possible mechanism involved in the action of these extracts. Both extracts produced a significant drop $(\mathrm{P}<0.05)$ in plasma triglycerides, total cholesterol, cholesterol (VLDL) and cholesterol (LDL), however, an increase in cholesterol (HDL) was observed (P <0.05 ). The hypercholesterolemic regression test revealed a significant reduction $(\mathrm{P}<0.05)$ in the serum cholesterol level in the treated animals (Subbarao \& Ashok, 2011). This species shows promising potential for future herbal medicines used in the treatment of dyslipidemia and in the prevention of atherosclerosis. The authors reported that this action is possibly due to the tetranoterpenes and triterpenes found in this plant.

\section{Trichilia rubescens Oliv.}

We found a study in this time range. It should be noted that Trichilia rubescens Oliv. it is mostly found in regions of Tropical Africa, which may explain the limited number of studies. The action of the crude extracts (methanolic and ethyl acetate) of the leaves was evaluated. What the authors highlighted in this work was the strong cytotoxic action of both extracts against Plasmodium falciparum (IC50 $3.1 \mathrm{mg} / \mathrm{mL}$ ). After fractionation of the ethyl acetate extract, a significant antimalarial activity of the limonoids (trichirubins A and B) (IC50 $4 \mathrm{mg} / \mathrm{mL}$ ) was observed. Therefore, they were identified as the possible responsible for this antimalarial action (Krief et al., 2006). The authors suggested investigative studies regarding an antiinflammatory action, due to its popular use for this purpose. 


\section{Trichilia lepidota Mart.}

For this species, a study was found using hexanic and methanolic extracts of the leaves and fractions of limonoids of the fruit were tested for their cytotoxic action against leukemic cell lines MOLT-4 and U937. The protolimonids 6 isolated from this plant were more toxic (IC50 $9.3 \mathrm{mg} / \mathrm{mL}$ ) compared to both strains tested. As for the extracts, the hexanic (IC50 $62.5 \mathrm{mg} / \mathrm{mL}$ ) exhibited the most significant cytotoxic activity against both strains (Terra et al., 2013). It is important to note that protolimonids are proven to be toxic to tumor cells. Thus, this plant is promising for the development of new drugs with antineoplastic action.

Although 47 species of Trichilia occur in Brazil (Pennington, Styles \& Taylor, 1981), in a period of 15 years, only three species were investigated regarding their therapeutic activity (Trichilia catigua, Trichilia lepidota and Trichilia silvatica) showing that, as reported by David, Wolfender and Dias (2014) although popular knowledge has contributed significantly to the understanding of the effects of many medicinal plants, other plants are still little known as to their active principles, mechanism of action and toxicity, we can see this with the plants of the genus Trichilia, thus justifying further studies to scientifically validate the effectiveness of its constituents.

Medicinal plants are important for the synthesis of new drugs. In this context, the genus Trichilia is promising, due to its biological activities and secondary metabolites. However, we observed that many species of this genus have reduced numbers of studies. Perhaps this fact can be explained due to their restricted geographical location. Disseminating the knowledge acquired about these plants is indeed important for future research.

\section{Conclusion}

It was shown through the studies mentioned in this review that the genus Trichilia is promising for the treatment of numerous diseases. The studies mentioned here also reveal that plants of this genus can be important allies in combating pathogens resistant to conventional drugs. As we pointed out in this study, species of the genus Trichilia still have a lot to offer us in combating and even preventing diseases such as diabetes, atherosclerosis, fungal, bacterial, neoplastic and neurodegenerative diseases. Many of these diseases have yet to be cured. However, many species of the genus Trichilia are little known, which results in little research on their therapeutic properties. This review highlights the main therapeutic applications found in studies in the literature with plants of the genus Trichilia. It demonstrates its great biotechnological potential, in order to stimulate further studies on its employability in the development of herbal medicines for the treatment of numerous diseases that affect humans.

\section{Acknowledgments}

The authors would like to thank the Coordination for the Improvement of Higher Education Personnel (CAPES) and Universidade Federal Fluminense for the support provided for the development of this research.

\section{Authors' Contributions}

These authors contributed equally. Lusinalva Leonardo da Silva, Renata de Almeida, Fabiana Teixeira e Silva and Maurício Afonso Verícimo designed the research, analyzed data and wrote the manuscript.

\section{References}

Applequist, W. L., Brinckmann, J. A., Cunningham, A. B., Hart, R. E., Heinrich, M., Katerere, D. R., \& Van Andel, T. (2020). Scientists warning on climate change and medicinal plants. Planta Medica, 86(1), 10-18. https://doi.org/10.1055/a-1041-3406.

Ben, I. O., Woode, E., Koffuor, G. A., \& Asiamah, E. A. (2016). Anti-anaphylactic effects of Trichilia monadelpha (Thonn.) J. J. De Wilde extracts on rodent models of anaphylaxis. Research in pharmaceutical sciences, 11(5), 397-404. https://doi.org/10.4103/1735-5362.192491. 
Bernardo, J., Ferreres, F., Gil-Izquierdo, Á., Videira, R. A., Valentão, P., Veiga, F., \& Andrade, P. B. (2018). In vitro multimodal-effect of Trichilia catigua A. Juss. (Meliaceae) bark aqueous extract in CNS targets. Journal of ethnopharmacology, 211, 247-255. https://doi.org/10.1016/j.jep.2017.09.039.

Campos, M. M., Fernandes, E. S., Ferreira, J., Santos, A. R., \& Calixto, J. B. (2005). Antidepressant-like effects of Trichilia catigua (Catuaba) extract: evidence for dopaminergic-mediated mechanisms. Psychopharmacology, 182(1), 45-53. https://doi.org/10.1007/s00213-005-0052-1.

Da Silva, J. V., Dos Santos, R. C., Júnior, P., Pederiva, M., do Carmo Vieira, M., Kassuya, C., Cardoso, C., Pereira, Z. V., Ruiz, A., Foglio, M. A., De Carvalho, J. E., \& Formagio, A. (2018). Anti-inflammatory, Antioxidant and Antiproliferative Activities from Trichilia silvatica (C.DC). Current pharmaceutical biotechnology, 19(12), 973-981. https://doi.org/10.2174/1389201020666181123121817.

Da Silva, M. F., Ottor, R. G., \& David, D. (1984). Evolution of limonoids in Meliaceae. Biochemical Systematics and Ecology, 12(3), 299-310. https://doi.org/10.1016/0305-1978(84)90053-X.

David, B., Wolfender, J., \& Dias, D. (2014). The pharmaceutical industry and natural products: historical status and new trends. Phytochemistry Reviews, 14, 299-315. https://doi.org/10.1007/s11101-014-9367-z.

do Nascimento Bonato Panizzon, C. P., de Miranda Neto, M. H., Ramalho, F. V., Longhini, R., de Mello, J., \& Zanoni, J. N. (2019). Ethyl Acetate Fraction from Trichilia catigua Confers Partial Neuroprotection in Components of the Enteric Innervation of the Jejunum in Diabetic Rats. Cellular physiology and biochemistry : international journal of experimental cellular physiology, biochemistry, and pharmacology, 53(1), 76-86. https://doi.org/10.33594/000000122.

do Nascimento Bonato Panizzon, C. P., de Miranda Neto, M. H., Ramalho, F. V., Longhini, R., de Mello, J., \& Zanoni, J. N. (2019). Ethyl Acetate Fraction from Trichilia catigua Confers Partial Neuroprotection in Components of the Enteric Innervation of the Jejunum in Diabetic Rats. Cellular physiology and biochemistry : international journal of experimental cellular physiology, biochemistry, and pharmacology, 53(1), 76-86. https://doi.org/10.33594/000000122.

Eldeen, I. M., Elgorashi, E. E., \& van Staden, J. (2005). Antibacterial, anti-inflammatory, anti-cholinesterase and mutagenic effects of extracts obtained from some trees used in South African traditional medicine. Journal of ethnopharmacology, 102(3), 457-464. https://doi.org/10.1016/j.jep.2005.08.049.

Eldin, S., \& Dunford, A. Phytotherapy in primary health care. (2011). São Paulo: Manole; P.163. https://doi.org/https://doi.org/10.1590/S0102$311 \times 2007000600021$.

Espada, S. F., Faccin-Galhardi, L. C., Rincao, V. P., Bernardi, A. L., Lopes, N., Longhini, R., de Mello, J. C., Linhares, R. E., \& Nozawa, C. (2015). Antiviral Activity of Trichilia catigua Bark Extracts for Herpesvirus and Poliovirus. Current pharmaceutical biotechnology, 16(8), 724-732. https://doi.org/10.2174/1389201016666150505125235.

Estevão, L. R. M., Mendonça, F. S., Baratella-Evênci, L., Simões, R. S., Barros, M. E. G., Arantes, R. M. E., Rachid, M. A., \& Evêncio-Neto, J. (2013). Effects of aroeira (Schinus terebinthifoliu Raddi) oil on cutaneous wound healing in rats. Acta Cirúrgica Brasileira, 28(3), 202-209. https://doi.org/10.1590/s0102-86502013000300008.

Geyid, A., Abebe, D., Debella, A., Makonnen, Z., Aberra, F., Teka, F., Kebede, T., Urga, K., Yersaw, K., Biza, T., Mariam, B. H., \& Guta, M. (2005). Screening of some medicinal plants of Ethiopia for their anti-microbial properties and chemical profiles. Journal of ethnopharmacology, 97(3), 421-427. https://doi.org/10.1016/j.jep.2004.08.021.

Germanò, M. P., D'Angelo, V., Sanogo, R., Catania, S., Alma, R., De Pasquale, R., \& Bisignano, G. (2005). Hepatoprotective and antibacterial effects of extracts from Trichilia emetica Vahl. (Meliaceae). Journal of ethnopharmacology, 96(1-2), 227-232. https://doi.org/10.1016/j.jep.2004.09.011.

Godinho, J., de Oliveira, R., de Sa-Nakanishi, A. B., Bacarin, C. C., Huzita, C. H., Longhini, R., Mello, J., Nakamura, C. V., Previdelli, I. S., Dal Molin Ribeiro, M. H., \& Milani, H. (2018). Ethyl-acetate fraction of Trichilia catigua restores long-term retrograde memory and reduces oxidative stress and inflammation after global cerebral ischemia in rats. Behavioural brain research, 337, 173-182. https://doi.org/10.1016/j.bbr.2017.08.050.

Godinho, J., de Sa-Nakanishi, A. B., Moreira, L. S., de Oliveira, R., Huzita, C. H., Mello, J., da Silva, A., Nakamura, C. V., Previdelli, I. S., Ribeiro, M., \& Milani, H. (2018). Ethyl-acetate fraction of Trichilia catigua protects against oxidative stress and neuroinflammation after cerebral ischemia/reperfusion. Journal of ethnopharmacology, 221, 109-118. https://doi.org/10.1016/j.jep.2018.04.018.

Gomes, R. M., de Paulo, L. F., Bonato Panizzon, C., Neves, C. Q., Cordeiro, B. C., Zanoni, J. N., Francisco, F. A., Piovan, S., de Freitas Mathias, P. C., Longhini, R., de Mello, J., de Oliveira, J. C., Pedrino, G. R., da Silva Reis, A. A., Cecchini, A. L., \& Marçal Natali, M. R. (2017). Anti-Diabetic Effects of the Ethyl-Acetate Fraction of Trichilia catigua in Streptozo-tocin-Induced Type 1 Diabetic Rats. Cellular physiology and biochemistry: international journal of experimental cellular physiology, biochemistry, and pharmacology, 42(3), 1087-1097. https://doi.org/10.1159/000478761.

Hayouni, E. A., Miled, K., Boubaker, S., Bellasfar, Z., Abedrabba, M., Iwaski, H., Oku, H., Matsui, T., Limam, F., \& Hamdi, M. (2011). Hydroalcoholic extract based-ointment from Punica granatum L. peels with enhanced in vivo healing potential on dermal wounds. Phytomedicine: international journal of phytotherapy and phytopharmacology, 18(11), 976-984. https://doi.org/10.1016/j.phymed.2011.02.011.

Hernandez, E., González, B., Díaz, A., González, M., Morris, H. J., Delgado, L., \& Martínez, C. E .M. (2013). Ethnopharmacological evaluation of Trichilia hirta L. as anticancer source in traditional medicine of Santiago de Cuba. Bol. latinoam. Caribe de plantas medicinales y aromáticas, 12, 176-185. https://corpus.co.id/en/lil-722790.

Kamdem, J. P., Stefanello, S. T., Boligon, A. A., Wagner, C., Kade, I. J., Pereira, R. P., Preste, A., Roos, D. H., Waczuk, E. P., Appel, A. S., Athayde, M. L., Souza, D. O., \& Rocha, J. B. (2012). In vitro antioxidant activity of stem bark of Trichilia catigua Adr. Juss. Acta pharmaceutica (Zagreb, Croatia), 62(3), 371-382. https://doi.org/10.2478/v10007-012-0026-x.

Konaté, K., Yomalan, K., Sytar, O., Zerbo, P., Brestic, M., Patrick, V. D., Gagniuc, P., \& Barro, N. (2014). Free Radicals Scavenging Capacity, Antidiabetic and Antihypertensive Activities of Flavonoid-Rich Fractions from Leaves of Trichilia emetica and Opilia amentacea in an Animal Model of Type 2 Diabetes Mellitus. Evidence-based complementary and alternative medicine: eCAM, 2014, 867075. https://doi.org/10.1155/2014/867075. 
Krief, S., Huffman, M. A., Sévenet, T., Hladik, C. M., Grellier, P., Loiseau, P. M., \& Wrangham, R. W. (2006). Bioactive properties of plant species ingested by chimpanzees (Pan troglodytes schweinfurthii) in the Kibale National Park, Uganda. American journal of primatology, 68(1), 51-71. https://doi.org/10.1002/ajp.20206.

Kuglerova, M., Halamova, K., Kokoska, L., Van Damme, P., \& Grade, J. (2007). Antimicrobial activity of Ugandan Medicinal Plants. Planta Medica, 73, 113. https://doi.org/10.1055/s-2007-986895.

Martins, N. O., de Brito, I. M., Araújo, S., Negri, G., Carlini, E. A., \& Mendes, F. R. (2018). Antioxidant, anticholinesterase and antifatigue effects of Trichilia catigua (catuaba). BMC complementary and alternative medicine, 18(1), 172. https://doi.org/10.1186/s12906-018-2222-9.

Naidoo, D., van Vuuren, S. F., van Zyl, R. L., \& de Wet, H. (2013). Plants traditionally used individually and in combination to treat sexually transmitted infections in northern Maputaland, South Africa: antimicrobial activity and cytotoxicity. Journal of ethnopharmacology, 149(3), 656-667. https://doi.org/10.1016/j.jep.2013.07.018.

Opawale, B., Oyetayo, A., \& Agbaje, R. (2015). Phytochemical Screening, Antifungal and Cytotoxic Activities of Trichilia heudelotii Planc (Harm). International Journal of Sciences: Basic and Applied Research, 24(6), 267-276. https://corpus.co.id/en/56442254.

Pennington, T. D., Styles, B. S., \& Taylor, D. A. H. (1981). Meliaceae. Flora Neotropica, 28, 1-470. https://doi.org/10.11646/phytotaxa.259.1.5.

Pennington, T. D., \& Styles, B. S. (1975). A generic monograph of Meliaceae. Blumea, 22, 419-540. https://doi.org/10.2307/4110009

Pennington, T. D. (2016). Systematic treatment of American Trichilia (Meliaceae). Phytotaxa, 259. https://doi.org/10.11646/phytotaxa.259.1.5.

Ramírez, M. C., Toscano, R. A., Arnason, J., Omar, S., Cerda-Garcia-Rojas, C. M., \& Mata R. (2000). Structure, conformation and absolute configuration of new antifeedant dolabellanes from Trichilia trifolia. Tetrahedron, 56(29), 5085-5091. https://doi.org/10.1016/S0040-4020(00)00423-3.

Ritter, M. R., Tempesta de, O. M., Makimori, R. Y., Sereia, A. L., Simionato, A. S., Chierrito, D., Galdino, A. F., Gonçalves de, O. A., Brentan da, S. D., Novello, C. R., Cristina de, M. D., Benedito Prado, D. F., \& João Carlos Palazzo de, M. (2019). Dimeric glycosylated flavan-3-ol and antimicrobial in vitro evaluation of Trichilia catigua extracts. Natural product research, 1-8. Advance online publication. https://doi.org/10.1080/14786419.2019.1698569.

Silva, M.F., Gottlieb, O., \& Dreyer, D.L. (1984). Evolution of limonoids in the Meliaceae. Biochemical Systematics and Ecology, 12, 299-310. https://doi.org/10.1016/0305-1978(84)90053-X.

Stefano, M. V., Calazans, L. S., \& Sakuragui, C. M. (2014). Meliaceae In: List of Species of Flora of Brazil. Botanical Garden of Rio de Janeiro. http://floradobrasil.jbrj.gov.br/jabot/floradobrasil/FB162.

Schenkel, E. P. (1995). Watch your medications. Medicinal plants, teas and herbal medicines. Saga, Deluzzata.

Sosa, E. H., Duharte, A. B., Portuondo, D., Tamayo, O. V., Mora, González, N., Morris Quevedo, H. J., \& Manrique, C. E. (2010). Immunorestorative in immunosuppressed Balb/c mice and cytotoxic activity of water extract from Trichilia hirta root. Boletín Latinoamericano y del Caribe de Plantas Medicinales y Aromáticas, 9(6), 457-464. https://corpus.co.id/en/lil-644984.

Sosa, E. H., Castejón, Y. M., Duharte, A. B., Portuondo, D., Tamayo, V., Quevedo, H. J., \& Manrique, C. E. (2011). Leukocyte-stimulating effect and phytochemical screening of Trichilia hirta extracts. Journal of medicinal food, 14(9), 1057-1059. https://doi.org/10.1089/jmf.2010.0166.

Sosa, E. H., Mora Gonzalez, N., Morris, Q., \& Humberto, J. (2013). Actividad citotóxica de extractos acuosos de hojas de Trichilia hirta sobre células tumorales humanas. Cuban Journal of Biomedical Research, 32(1), 93-101. https://corpus.co.id/en/lil-673097.

Subbarao, P. G., \& Ashok, P. (2011). Antihyperlipidemic effect of Trichilia connaroides in hypercholesterolemic rats and its possible mechanism. Journal of pharmacy \& bioallied sciences, 3(2), 230-235. https://doi.org/10.4103/0975-7406.80777.

Taciany Bonassoli, V., Micheli Chassot, J., Longhini, R., Milani, H., Mello, J. C., \& de Oliveira, R. M. (2012). Subchronic administration of Trichilia catigua ethyl-acetate fraction promotes antidepressant-like effects and increases hippocampal cell proliferation in mice. Journal of ethnopharmacology, 143(1), 179184. https://doi.org/10.1016/j.jep.2012.06.021.

Terra, W., Vieira, I. J., Braz-Filho, R., Freitas, W. R., Kanashiro, M. M., \& Torres, M. C. (2013). Lepidotrichilins A and B, new protolimonoids with cytotoxic activity from Trichilia lepidota (Meliaceae). Molecules (Basel, Switzerland), 18(10), 12180-12191. https://doi.org/10.3390/molecules181012180.

Truiti, M. T., Soares, L., Longhini, R., Milani, H., Nakamura, C. V., Mello, J. C., \& de Oliveira, R. M. (2015). Trichilia catigua ethyl-acetate fraction protects against cognitive impairments and hippocampal cell death induced by bilateral common carotid occlusion in mice. Journal of ethnopharmacology, 172, 232237. https://doi.org/10.1016/j.jep.2015.05.060.

Viana, A. F., Maciel, I. S., Motta, E. M., Leal, P. C., Pianowski, L., Campos, M. M., \& Calixto, J. B. (2011). Antinociceptive Activity of Trichilia catigua Hydroalcoholic Extract: New Evidence on Its Dopaminergic Effects. Evidence-based complementary and alternative medicine: eCAM, 2011 , 120820. https://doi.org/10.1093/ecam/nep144.

Vicentini, F. A., Barbosa, M., Fortunato, M. C., Amado, C., Comar, J. F., Longhini, R., de Mello, J., \& Natali, M. (2018). Treatment with Trichilia catigua ethyl-acetate fraction improves healing and reduces oxidative stress in TNBS-induced colitis in rats. Biomedicine \& pharmacotherapy $=$ Biomedecine $\&$ pharmacotherapie, 107, 194-202. https://doi.org/10.1016/j.biopha.2018.07.160. 